\title{
A BIOLOGICAL PROCESS SUBJECT TO NONCOMPETITIVE SUBSTRATE INHIBITION IN A GENERALIZED FLOW REACTOR
}

\begin{abstract}
M. I. NELSON ${ }^{\otimes 1}$, T. NICHOLLS ${ }^{1}$ and N. HAMZAH ${ }^{2}$
(Received 8 October, 2012; revised 3 March, 2013; first published online 26 July, 2013)

Abstract

We analyse the steady-state operation of a continuous flow bioreactor in which the biochemical reaction is governed by noncompetitive substrate inhibition (Andrews kinetics). A generalized reactor model is used in which the well-stirred bioreactor and the idealized membrane bioreactor are special cases. As generic properties of systems subject to substrate inhibition have been obtained by other authors, we discuss reaction engineering features specific to Andrews kinetics.
\end{abstract}

2010 Mathematics subject classification: 92C45.

Keywords and phrases: bioreactor, kinetics, stirred tank, substrate inhibition.

\section{Introduction}

A continuous stirred flow bioreactor is a well-stirred vessel containing microorganisms $(X)$ through which a substrate $(S)$ flows at a continuous rate. The microorganisms grow in the vessel through the consumption of the substrate to produce more microorganisms and products. Unused substrate, microorganisms and products flow out of the reactor at the same rate at which the feed is admitted. In membrane bioreactors, a permeable membrane is used which physically retains microorganisms inside the reactor whilst allowing the substrate and products to leave. Entrapping the microorganisms in this manner increases their concentration and results in a greater conversion of the substrate. This allows for a more rapid and efficient process.

The standard way to model the specific growth rate of microorganisms $(\mu)$ growing on a limiting substrate is to use the Monod model. This is given by

$$
\mu(S)=\frac{\mu_{m} S}{K_{s}+S}
$$

\footnotetext{
${ }^{1}$ School of Mathematics and Applied Statistics, University of Wollongong, Wollongong, NSW 2522, Australia; e-mail: nelsonm@member.ams.org.

${ }^{2}$ Department of Mathematics, Faculty of Science, Universiti Brunei Darussalam, Brunei Darussalam.

(C) Australian Mathematical Society 2013, Serial-fee code 1446-1811/2013 \$16.00
} 
In this equation, $\mu_{m}$ is the maximum value of the specific growth rate, obtained in the limit as $S \rightarrow \infty$, and $K_{s}$ is the Monod constant, the substrate concentration at which the specific growth rate obtains half its maximum value. The Monod model is an increasing function of the substrate concentration: the more substrate available, the quicker the microorganisms grow.

For processes subject to substrate inhibition, the specific growth rate is an increasing (decreasing) function at low (high) values of the substrate concentration, that is,

$$
\frac{d \mu}{d S} \begin{cases}>0 & \text { if } S<S_{\mathrm{cr}}, \\ <0 & \text { if } S>S_{\mathrm{cr}}\end{cases}
$$

In this equation, $S_{\mathrm{cr}}$ is the value of the substrate concentration at which substrate inhibition "sets in", that is, the transition point at which the specific growth rate changes from an increasing function of the substrate concentration to a decreasing function of the substrate concentration.

Substrate inhibition is common in aerobic fermentation processes, the biological treatment of industrial wastewaters and wastes, and when the substrate is toxic to the microorganism. A simple modification to the Monod model which is widely used to represent growth kinetics subject to substrate inhibition is the Andrews model [1]

$$
\mu(S)=\frac{\mu_{m} S}{K_{S}+S+S^{2} / K_{I}} .
$$

In the Andrews model,

$$
S_{\mathrm{cr}}=\sqrt{K_{I} K_{S}}
$$

Note that in equation (1.2) the parameters $\mu_{m}$ and $K_{s}$ no longer have any intrinsic kinetic meaning. Recent examples of biological process that have been identified as being governed by Andrew kinetics include the removal of diethylketone from aqueous solution by an Arthrobacter viscosus culture [4], the dechlorination of water containing high concentrations of tetrachloroethene by two dechlorinating biomass strains [7] and the growth of of the oleaginous fungus Mortierella isabellina upon sweet sorghum extract [5].

\section{Equations and literature review}

In Section 2.1 we give equations for the concentration of microorganisms and substrate within a perfectly stirred, well-aerated bioreactor. In Section 2.2 the equations are scaled. In Section 2.3 we provide a literature review.

The parameter $\beta, 0 \leq \beta \leq 1$, defines the reactor configuration. The cases $\beta=0$ and $\beta=1$ represent an idealized membrane reactor and a well-stirred reactor, respectively. The case $0<\beta<1$ includes: a non-idealized membrane reactor; a fixed-bed biological process with a large recirculation rate and with biomass detachment; a perfusion bioreactor incorporating cell bleed; and a well-stirred reactor, attached to a settling unit. 
2.1. The dimensional model The model equations are

$$
\begin{aligned}
& V \frac{d S}{d t}=F\left(S_{0}-S\right)-\frac{\mu(S)}{\alpha_{s}} V X, \\
& V \frac{d X}{d t}=-\beta F X+\mu(S) V X-V k_{d} X .
\end{aligned}
$$

The specific growth rate is given by by the Andrews model (1.2) and we also define the residence time

$$
\tau=\frac{V}{F} .
$$

In these equations the dependent variables $S$ and $X$ denote the concentration of substrate and microorganisms, respectively, whilst the independent variable $t$ denotes time. The other parameters are: $F$, the flow rate through the reactor; $K_{I}$, the substrate inhibition constant; $K_{s}$, the Monod parameter; $S_{0}$, the concentration of substrate in the feed; $V$, the volume of the reactor; $k_{d}$, the decay coefficient of the microorganisms; $\alpha_{s}$, the yield factor (the ratio of the increase in the "concentration" of the microorganisms to the corresponding decrease in the "concentration" of the substrate); $\beta$, the reactor configuration parameter; $\mu_{m}$, a kinetic parameter; and $\mu_{S}$, the specific growth rate. The units of the terms appearing in equations (2.1)-(2.3) are defined in Appendix A. Monod kinetics are recovered in the limit as the substrate inhibition constant $\left(K_{I}\right)$ becomes infinite.

For a specific biological culture and a particular set of environmental conditions, the parameters $K_{I}, K_{S}, k_{d}, \alpha_{s}$ and $\mu_{\max }$ are fixed. The parameters that can be varied are $S_{0}$ and $\tau$. The main experimental control parameter, that is, the primary bifurcation parameter, is the residence time $\tau$.

2.2. The dimensionless model By introducing the dimensionless variables defined in Appendix A, the dimensional model (2.1)-(2.2) can be written in the dimensionless form

$$
\begin{aligned}
& \frac{d S^{*}}{d t^{*}}=\frac{1}{\tau^{*}}\left(S_{0}^{*}-S^{*}\right)-\frac{S^{*} X^{*}}{1+S^{*}+\gamma S^{*^{2}}}, \\
& \frac{d X^{*}}{d t^{*}}=-\frac{\beta}{\tau^{*}} X^{*}+\frac{S^{*} X^{*}}{1+S^{*}+\gamma S^{2}}-k_{d}^{*} X^{*},
\end{aligned}
$$

where the parameter groups are defined in Appendix A. A low value for the dimensionless substrate inhibition parameter $\gamma$ indicates that the biomass is not sensitive to substrate inhibition. The initial conditions associated with equations (2.4) and (2.5) must be physically meaningful: $S^{*}\left(t^{*}=0\right) \geq 0$ and $X^{*}\left(t^{*}=0\right) \geq 0$.

From now on we assume that the dimensionless residence time, the dimensionless feed concentration, the dimensionless substrate inhibition constant and the reactor parameter are all strictly positive $\left(\tau^{*}>0, S_{0}^{*}>0, \gamma>0\right.$ and $\beta>0$, respectively). The case $\beta=0$ is not considered because if $\beta=0$ then the steady-state substrate concentration is independent of the residence time. 
Finally, we note that the maximum value of the function

$$
\mu\left(S^{*}\right)=\frac{S^{*}}{1+S^{*}+\gamma S^{* 2}}
$$

occurs when

$$
S^{*}=S_{\mathrm{cr}}^{*}=\gamma^{-1 / 2}
$$

and is given by

$$
\mu\left(S^{*}\right)_{\max }=\frac{1}{1+2 \sqrt{\gamma}} .
$$

2.3. Literature review Andrews [1] investigated the model (2.1)-(2.2) in a flow reactor $(\beta=1)$ with the assumption that the microorganisms do not die $\left(k_{d}=0\right)$. The steady-state solution along the no-washout branch and the critical value of the residence time at which the washout solution loses stability were both identified. Andrews noted that at a fixed value for the residence time an increase in the feed concentration of the inhibitory substrate may result in process instability: the nowashout solution branch loses stability, whilst the washout solution branch gains stability.

Bush and Cook [2] investigated (2.1)-(2.2), replacing the Andrews function by a general inhibition function $\mu(S)$ satisfying (1.1). They showed that when there are two steady-state solutions with substrate components $0<S_{1}<S_{2}<S_{0}$, the steadystate solutions corresponding to $S_{1}$ and $S_{2}$ are stable and unstable, respectively. Furthermore, there are no steady-state solutions with purely imaginary eigenvalues. These results apply to the case $0<\beta<1$ and $k_{d} \neq 0$.

For the case $\beta=1$ and $k_{d}=0$, Bush and Cook reduced the system to a single differential equation and characterized the global behaviour of the model. (This reduction is possible when $\beta=1$ and $k_{d}=0$ as all solutions converge to a onedimensional manifold.) They explained how shock overloading, a temporary large increase in the feed concentration followed by a return to the normal input concentration, and shock underloading, a temporary large decrease in the feed concentration followed by a return to the normal input concentration, can lead to system failure.

The model (2.1)-(2.2) has been generalized in the mathematics literature to a system of $N$ microorganisms competing for a substrate [3,9-11, 13]. These papers consider a general class of specific growth-rate functions which include monotone increasing functions and uptake functions exhibiting substrate inhibition. It is assumed that along the no-washout solution branch there are at most two solutions with substrate component satisfying $0<S_{1}<S_{2}<S_{0}$. The primary goal of these investigations is to establish that at most one species survives. This result, the principle of competitive exclusion, is established by constructing an appropriate Lyapunov function. It is usually not stated whether inhibition functions commonly used in chemical engineering satisfy the conditions required in the construction of the Lyapunov function. 
The initial work on this problem is due to Butler and Wolkowicz [3], who considered the case $\beta=1, k_{d}=0$. Their work has been extended to the case of distinct removal rates (equivalent to $0<\beta \leq 1, k_{d}>0$ ) by Wolkowicz and $\mathrm{Lu}$ [13] and $\mathrm{Li}$ [9]. The model with distinct removal rates has been extended to include a time delay in the nutrient conversion process [12] and to the case when the yield, $\alpha_{s}$ in equation (2.1), is a function of the substrate concentration $[10,11]$.

Fiedler and Hsu [6] developed a higher-dimensional Bendixson-Dulac type exclusion principle, establishing conditions excluding (positive) periodic solutions in the chemostat with a single nutrient and $N$ competing species. Their results apply to both the cases of equal and unequal removal rates. The growth-rate functions are allowed to be non-monotone, although some conditions are required.

Although much is known about the general behaviour of systems subject to substrate inhibition, there has been little discussion about the specific behaviour when the Andrews growth rate law (1.2) is used. The aim of this paper is to investigate the steady-state behaviour of a biological process subject to Andrews inhibition in a generalized bioreactor model.

\section{Results}

The global behaviour of the system (2.4)-(2.5) is discussed in Section 3.1. In Section 3.2 the steady-state solution branches are given and the condition for the nowashout solution branch to be physically meaningful is stated. The local stability of the steady-state solutions is determined in Section 3.3. In Section 3.4 asymptotic solutions are presented for the cases of residence times just above the washout value $\left(\tau^{*}-\tau_{\mathrm{tr}}^{*} \ll 1\right)$ and large residence times $\left(\tau^{*} \gg 1\right)$.

3.1. Global behaviour In this section we state some global results regarding the system (2.4)-(2.5). For this system the region $\mathcal{R}$ defined by

$$
0 \leq S^{*} \leq S_{0}^{*}, \quad 0 \leq X^{*} \leq \frac{S_{0}^{*}}{\mathcal{M}}-S^{*}, \quad \mathcal{M}=\min \left(1, \beta+k_{d}^{*} \tau^{*}\right),
$$

is positively invariant [13, Lemma 2.1]. This means that if an initial condition is either inside or on the boundary of the region $\mathcal{R}$ then the corresponding solution of the system cannot leave this region for all values of time with $t^{*} \geq 0$. Furthermore, the invariant region is exponentially attracting for physically meaningful solutions starting outside the invariant region [13, Lemma 2.1]. Thus from now on we are free to only consider initial conditions within the invariant region.

The system (2.4)-(2.5) cannot have limit-cycle solutions. This is shown using Dulac's test [8, Ch. 3, Exercise 23] using the function $\rho=1 /\left(S^{*} X^{*}\right)$. As noted in Section 2.3, Bush and Cook [2] showed that the model (2.1)-(2.2) with a general specific growth rate satisfying (1.1) cannot have a steady-state solution with purely imaginary eigenvalues, that is, a Hopf bifurcation cannot occur. Our result showing the non-existence of limit cycles provides an alternative proof of this for the specific choice of the Andrews inhibition model (1.2). 
In Appendix B we identify conditions under which it is guaranteed that

$$
\lim _{t^{*} \rightarrow \infty} X^{*}=0 .
$$

In particular, this is always true if the decay parameter is sufficiently high:

$$
k_{d}^{*} \geq k_{d, \max , 1}=\frac{1}{1+2 \sqrt{\gamma}} .
$$

Consequently, from now we assume that

$$
k_{d}^{*}=\frac{1}{1+2 \sqrt{\gamma}}<1 .
$$

3.2. Steady-state solution branches In this section we find the steady-state solutions of the system (2.4)-(2.5) and characterize when they are physically meaningful.

The steady-state solutions are given by

$$
\begin{aligned}
\text { washout branch: }\left(S^{*}, X^{*}\right) & =\left(S_{0}^{*}, 0\right), \\
\text { no-washout branch: }\left(S^{*}, X^{*}\right) & =\left(\hat{S^{*}}, \frac{S_{0}^{*}-\hat{S}^{*}}{\beta+k_{d}^{*} \tau^{*}}\right),
\end{aligned}
$$

where $\hat{S^{*}}$ is given by

$$
\hat{S^{*}}=\frac{b \pm \sqrt{b^{2}-4 a c}}{2 a}, \quad a=\left(\beta+k_{d}^{*} \tau^{*}\right) \gamma, \quad b=\left(1-k_{d}^{*}\right) \tau^{*}-\beta, \quad c=\left(\beta+k_{d}^{*} \tau^{*}\right)
$$

In Appendix $\mathrm{C}$ we show that solutions of (3.3) are only positive for

$$
\tau^{*} \geq \tau_{\max }^{*}=\frac{(1+2 \sqrt{\gamma}) \beta}{1-(1+2 \sqrt{\gamma}) k_{d}^{*}} .
$$

Before discussing the structure of the steady-state diagram we list a few useful observations.

(i) From (3.2) the no-washout branch is only physically meaningful when $0<\hat{S^{*}}<$ $S_{0}^{*}$.

(ii) When $\tau^{*}=\tau_{\max }^{*}$ we have $b^{2}-4 a c=0$ and (3.3) has exactly one solution,

$$
\hat{S}_{1}^{*}=\gamma^{-1 / 2} \text {. }
$$

(iii) When $\tau^{*}>\tau_{\max }^{*}$ we have $b^{2}-4 a c>0$ and (3.3) has two solutions. We denote these by $\hat{S}_{+}^{*}$ and $\hat{S}_{-}^{*}$, corresponding to the positive and negative square root sign in (3.3). 
(iv) Differentiating (3.3), we obtain

$$
\frac{d \hat{S}^{*}}{d \tau}=\frac{\beta \gamma}{2 a^{2}}\left[1 \pm \frac{b}{\sqrt{b^{2}-4 a c}}\right] .
$$

Thus for $\tau^{*}>\tau_{\max }^{*}$ the solution branch $\hat{S}_{+}^{*}$ is an increasing function of the residence time, whilst the solution branch $\hat{S}_{-}^{*}$ is a decreasing function of the residence time.

(v) From (3.3) the value of the residence time at which $\hat{S}^{*}=S_{0}^{*}$ is

$$
\tau^{*}=\tau_{\mathrm{tr}}^{*}=\frac{\beta\left(1+S_{0}^{*}+\gamma S_{0}^{2}\right)}{S_{0}^{*}-\left(1+S_{0}^{*}+\gamma S_{0}^{*^{2}}\right) k_{d}^{*}} .
$$

Note that the denominator of this expression is positive as by assumption

$$
k_{d}^{*}<\frac{S_{0}^{*}}{1+S_{0}^{*}+\gamma S_{0}^{*^{2}}} .
$$

We show in Appendix D that, when they are physically meaningful, $\tau_{\mathrm{tr}}^{*} \geq \tau_{\max }^{*}$ with equality only when $\gamma=1 / S_{0}^{*^{2}}$.

Assuming that inequality (3.1) holds, it follows from our preceding remarks that there are two generic steady-state diagrams and one non-generic steady-state diagram. These are described as follows:

Case 1. $\hat{S}_{1}^{*}=\gamma^{-1 / 2}>S_{0}^{*}$ (generic). The solution branch $\hat{S}_{+}^{*}$ is never physically meaningful as $\hat{S}_{+}^{*}>\hat{S}_{1}^{*}>S_{0}^{*}$. The solution branch $\hat{S}_{-}^{*}$ is not physically meaningful for $\tau_{\max }^{*}<\tau^{*}<\tau_{\mathrm{tr}}^{*}$ as over this parameter range $\hat{S}_{-}^{*}>S_{0}^{*}$. The solution branch $\hat{S}_{-}^{*}$ is physically meaningful for $\tau^{*}>\tau_{\text {tr }}^{*}$ as we have $S_{-}^{*}<S_{0}^{*}$. There is a transcritical bifurcation at $\tau^{*}=\tau_{\mathrm{tr}}^{*}$, where the solution branch $S_{-}^{*}$ intersects the washout solution branch, and a non-physical limit point at

$$
\left(S^{*}, X^{*}, \tau^{*}\right)=\left(\gamma^{-1 / 2}, \frac{S_{0}^{*}-\gamma^{-1 / 2}}{\beta+k_{d}^{*} \tau_{\max }^{*}}, \tau_{\max }^{*}\right) .
$$

Case 2. $\hat{S}_{1}^{*}=\gamma^{-1 / 2}=S_{0}^{*}$ (non-generic). The solution branch $\hat{S}_{+}^{*}$ is never physically meaningful because $\hat{S}_{+}^{*}>\hat{S}_{1}^{*}>S_{0}^{*}$. The solution branch $\hat{S_{-}^{*}}$ is physically meaningful for $\tau^{*}>\tau_{\mathrm{tr}}^{*}$. There is a pitchfork singularity at $\tau^{*}=\tau_{\mathrm{tr}}^{*}$ where the limit point on the no-washout solution branch occurs on the washout solution branch.

Case 3. $\hat{S}_{1}^{*}=\gamma^{-1 / 2}<S_{0}^{*}$ (generic). The solution branch $\hat{S}_{+}^{*}$ is physically meaningful for $\tau_{\max }^{*}<\tau^{*}<\tau_{\text {tr }}^{*}$. It is not physically meaningful for $\tau^{*}>\tau_{\text {tr }}^{*}$, where $\hat{S}_{+}^{*}>\hat{S}_{1}^{*}>S_{0}^{*}$. The solution branch $\hat{S}_{-}^{*}$ is physically meaningful for $\tau^{*}>\tau_{\mathrm{tr}}^{*}$. There is a transcritical bifurcation at $\tau^{*}=\tau_{\text {tr }}^{*}$, where the solution branch $S_{+}^{*}$ intersects the washout solution branch, and a physically meaningful limit point at

$$
\left(S^{*}, X^{*}, \tau^{*}\right)=\left(\gamma^{-1 / 2}, \frac{S_{0}^{*}-\gamma^{-1 / 2}}{\beta+k_{d}^{*} \tau_{\max }^{*}}, \tau_{\max }^{*}\right) .
$$






FIGURE 1. The variation of the dimensionless substrate concentration $S^{*}$ as a function of the dimensionless residence time $\tau^{*}$ and the inhibition constant in a well-stirred bioreactor. Stability is not shown. The circles and boxes indicate the locations of limit point and transcritical bifurcations, respectively. Parameter values: dimensionless death rate, $k_{d}^{*}=0.028$; dimensionless feed concentration, $S_{0}^{*}=1$; reactor parameter, $\beta=1$. The value of the dimensionless product inhibition constant is: (a) $\gamma=0.5$ (generic), (b) $\gamma=1.0$ (non-generic), (c) $\gamma=2.0$ (generic). Note that steady-state solutions for which $S^{*}>S_{0}^{*}=1$ are not physically meaningful; see (3.2).

Figure 1 illustrates the three steady-state diagrams. Lines (a) $\hat{S}_{1}^{*}=1.414>S_{0}^{*}$, (b) $\hat{S}_{1}^{*}=1.0=S_{0}^{*}$, and (c) $\hat{S}_{1}^{*}=0.707<S_{0}^{*}$ correspond to the first, second and third cases, respectively. In this figure the circles and boxes indicate the locations of limit point and transcritical bifurcations, respectively. Observe that in this figure $S_{0}^{*}=1$. Consequently, steady-state solutions for which $\hat{S}^{*}>S_{0}^{*}=1$ are not physically meaningful as the corresponding steady-state concentration of the microorganisms is negative; see (3.2).

\subsection{Stability of the steady-state solutions}

3.3.1. The washout solution The Jacobian matrix evaluated at the washout steadystate solution is given by

$$
J\left(S^{*}, X^{*}\right)=\left(\begin{array}{cc}
-\frac{1}{\tau^{*}} & -C \\
0 & -\frac{\beta}{\tau^{*}}+C-k_{d}^{*}
\end{array}\right), \quad C=\frac{S_{0}^{*}}{1+S_{0}^{*}+\gamma S_{0}^{*^{2}}} .
$$


The eigenvalues of this matrix are

$$
\lambda_{1}=-\frac{1}{\tau^{*}}<0, \quad \lambda_{2}=-\frac{\beta}{\tau^{*}}+\frac{S_{0}^{*}}{1+S_{0}^{*}+\gamma S_{0}^{*^{2}}}-k_{d}^{*} .
$$

It follows that the washout branch is always stable if

$$
k_{d}^{*} \geq k_{d, \max , 2}^{*}=\frac{S_{0}^{*}}{1+S_{0}^{*}+\gamma S_{0}^{*^{2}}} .
$$

In particular, the washout steady state is always stable when

$$
k_{d}^{*} \geq 1, \quad \text { which implies } k_{d} \geq \mu_{m} .
$$

This inequality makes sense "physically" because it shows that the washout steady state is always stable if the death rate is greater than, or equal to, the maximum growth rate.

If inequality (3.4) does not hold then the washout steady state is stable provided

$$
\tau^{*}<\tau_{\mathrm{tr}}^{*}=\frac{\left(1+S_{0}^{*}+\gamma S_{0}^{*^{2}}\right) \beta}{S_{0}^{*}-\left(1+S_{0}^{*}+\gamma S_{0}^{*^{2}}\right) k_{d}^{*}} .
$$

Differentiating (3.5) with respect to the inhibition parameter $\gamma$,

$$
\frac{d \tau_{\mathrm{tr}}^{*}}{d \gamma}=\frac{\beta S_{0}^{*^{3}}}{\left[S_{0}^{*}-\left(1+S_{0}^{*}+\gamma S_{0}^{*^{2}}\right) k_{d}^{*}\right]^{2}} .
$$

Thus, provided (3.4) does not hold, the value $\tau_{\mathrm{tr}}^{*}$ is an increasing function of the inhibition constant. (Once (3.4) holds the critical value is infinity.) Differentiating (3.5) with respect to the feed concentration gives

$$
\frac{d \tau_{\mathrm{tr}}^{*}}{d S_{0}^{*}}=\frac{\beta\left(\gamma S_{0}^{*}-1\right)}{\left[S_{0}^{*}-\left(1+S_{0}^{*}+\gamma S_{0}^{*^{2}}\right) k_{d}^{*}\right]^{2}} .
$$

Again, this equation is only valid as long as (3.4) does not hold. Thus if $\gamma S_{0}^{*}-1>0$ then $\tau_{\text {tr }}^{*}$ is an increasing function of the inhibition constant, that is, an increase in the feed concentration can lead to process instability. However, if $\gamma S_{0}^{*}-1<0$ then an increase in the feed concentration can stabilize the process. Results (3.6) and (3.7) were noted by Andrews [1] for the case $k_{d}^{*}=0$.

3.3.2. The no-washout solution The Jacobian matrix for the no-washout branch can be written in the form

$$
\begin{gathered}
J\left(S^{*}, X^{*},\right)=\left(\begin{array}{cc}
-\frac{1}{\tau^{*}}-A+B & -C \\
A-B & 0
\end{array}\right), \\
A=\frac{X^{*}}{1+S^{*}+\gamma S^{*^{2}}}, \quad B=\frac{X^{*} S^{*}\left(1+2 \gamma S^{*}\right)}{\left(1+S^{*}+\gamma S^{*^{2}}\right)^{2}}, \quad C=\frac{S^{*}}{1+S^{*}+\gamma S^{*^{2}}} .
\end{gathered}
$$


We have

$$
\text { trace } J=-\frac{1}{\tau^{*}}-(A-B), \quad \operatorname{det} J=C(A-B) .
$$

For physically meaningful solutions, $C>0$. Thus the condition for a physically meaningful solution to be stable is

$$
A>B \Rightarrow \operatorname{det} J>0, \quad \text { which implies trace } J<0 \text {. }
$$

After some algebra the condition $A>B$ gives $\gamma S^{*^{2}}<1$.

We consider the cases from Section 3.2.

Case 1. $\hat{S}_{1}^{*}=\gamma^{-1 / 2}>S_{0}^{*}$ (generic). The solution branch $\hat{S}_{+}^{*}$ is never physically meaningful, whilst the solution branch $\hat{S}_{-}^{*}$ is not physically meaningful for $\tau_{\max }^{*}<$ $\tau^{*}<\tau_{\mathrm{tr}}^{*}$. These cases can be ignored. The solution branch $\hat{S_{-}^{*}}$ is physically meaningful for $\tau^{*}>\tau_{\text {tr }}^{*}$ as we have $S_{-}^{*}<S_{0}^{*}$. Moreover,

$$
S_{-}^{*}<\hat{S}_{1}^{*}<\gamma^{-1 / 2} \quad \text { which implies } 1>\gamma S_{-}^{*^{2}} .
$$

Consequently, the solution branch $S_{-}^{*}$ is stable when it is physically meaningful.

Case 2. $\hat{S}_{1}^{*}=\gamma^{-1 / 2}=S_{0}^{*}$ (non-generic). The solution branch $\hat{S}_{+}^{*}$ is never physically meaningful, whilst the solution branch $S_{-}^{*}$ is always physically meaningful $\left(\tau^{*}>\tau_{\mathrm{tr}}^{*}\right)$. We have

$$
S_{-}^{*}<\hat{S}_{1}^{*}=\gamma^{-1 / 2} \quad \text { which implies } 1>\gamma S_{-}^{*^{2}} .
$$

Consequently, the solution branch $S_{-}^{*}$ is stable when it is physically meaningful.

Case 3. ${\hat{S^{*}}}_{1}^{*}=\gamma^{-1 / 2}<S_{0}^{*}$ (generic). The solution branch ${\hat{S_{+}^{*}}}^{*}$ is physically meaningful for $\tau_{\max }^{*}<\tau^{*}<\tau_{\mathrm{tr}}^{*}$. It is not physically meaningful for $\tau^{*}>\tau_{\mathrm{tr}}^{*}$, where $\hat{S_{+}^{*}}>\hat{S_{1}^{*}}>S_{0}^{*}$. We have

$$
S_{+}^{*}>\hat{S}_{1}^{*}=\gamma^{-1 / 2} \quad \text { which implies } 1>\gamma S_{+}^{*^{2}} .
$$

Thus the solution branch $S_{+}^{*}$ is unstable when it is physically meaningful. The solution branch $\hat{S}_{-}^{*}$ is always physically meaningful when it is defined, that is, provided that $\tau^{*}>\tau_{\max }^{*}$. We have

$$
S_{-}^{*}<\hat{S}_{1}^{*}=\gamma^{-1 / 2} \quad \text { which implies } 1>\gamma S_{-}^{*^{2}} .
$$

Consequently, the solution branch $S_{-}^{*}$ is stable when it is physically meaningful. Steady-state diagrams are shown in Figure 2. Only physically meaningful solutions have been plotted, that is, solutions for which $S^{*}>S_{0}^{*}$ are not shown.

3.4. Asymptotic solutions Asymptotic solutions are presented for the cases of residence times just above that at which the no-washout solution becomes physically meaningful and at large residence times. 


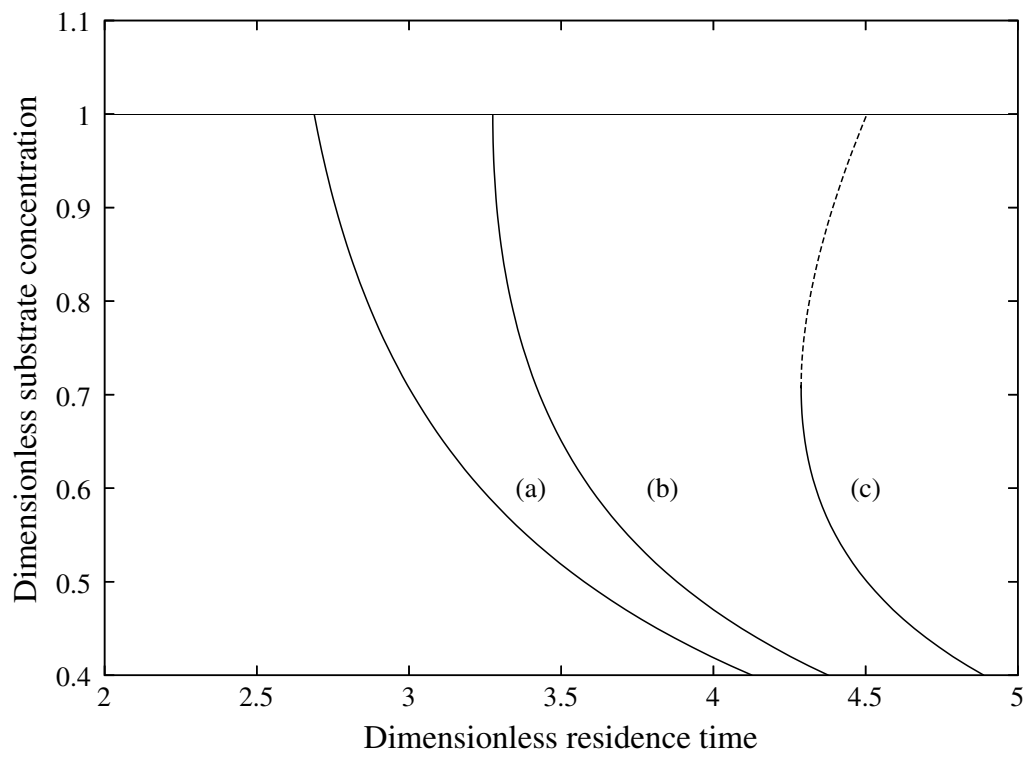

FIGURE 2. The two generic and one non-generic steady-state diagrams. Only physically meaningful solutions are shown. The stability of the washout solution changes at the bifurcation point. Parameter values as in Figure 1.

3.4.1. Residence times slightly higher than that at which the no-washout solution becomes physically meaningful $\left(\tau^{*}-\tau_{\mathrm{tr}}^{*} \ll 1\right)$ We have the following approximations to the stable steady-state solution.

For Case 1, line (a) in Figure 1, a transcritical bifurcation occurs at

$$
\tau^{*}=\tau_{\mathrm{tr}}^{*}=\frac{\left(1+S_{0}^{*}+\gamma S_{0}^{2}\right) \beta}{S_{0}^{*}-\left(1+S_{0}^{*}+\gamma S_{0}^{*^{2}}\right) k_{d}^{*}},
$$

where the washout solution branch and the no-washout solution branch intersect at the point

$$
\left(S^{*}, X^{*}, \tau^{*}\right)=\left(S_{0}^{*}, 0, \tau_{\mathrm{tr}}^{*}\right) .
$$

For values of the residence time slightly larger than the value at the bifurcation point, the concentrations along the no-washout branch are given by

$$
\begin{aligned}
& S_{-}^{*} \approx S_{0}^{*}-\frac{\left[-k_{d}^{*}+\left(1-k_{d}^{*}\right) S_{0}^{*}-k_{d}^{*} \gamma S_{0}^{*^{2}}\right]^{2}}{\left(1-\gamma S_{0}^{* 2}\right) \beta}\left(\tau^{*}-\tau_{\mathrm{tr}}^{*}\right)+O\left(\tau^{*}-\tau_{\mathrm{tr}}^{*}\right)^{2}, \\
& X^{*}=\frac{\left[-k_{d}^{*}+\left(1-k_{d}^{*}\right) S_{0}^{*}-k_{d}^{*} \gamma S_{0}^{* 2}\right]}{\left(1-\gamma S_{0}^{*^{2}}\right) \beta^{2} S_{0}^{*}}\left(\tau^{*}-\tau_{\mathrm{tr}}^{*}\right)+O\left(\tau^{*}-\tau_{\mathrm{tr}}^{*}\right)^{2} .
\end{aligned}
$$


For Case $2\left(\gamma^{-1 / 2}=S_{0}^{*}\right)$, line (b) in Figure 1, a pitchfork bifurcation occurs at

$$
\tau^{*}=\tau_{\mathrm{tr}}^{*}=\tau_{\max }^{*}=\frac{(1+2 \sqrt{\gamma}) \beta}{1-(1+2 \sqrt{\gamma}) k_{d}^{*}},
$$

where the washout solution branch and the no-washout solution branch intersect at the point

$$
\left(S^{*}, X^{*}, \tau^{*}\right)=\left(S_{0}^{*}, 0, \tau_{\mathrm{tr}}^{*}\right) .
$$

For values of the residence time slightly larger than the value at the bifurcation point, the concentrations along the no-washout branch are given by

$$
\begin{aligned}
S_{-}^{*} & \approx S_{0}^{*}-\frac{\left[S_{0}^{*} \beta\right]^{1 / 2}}{g \beta} A\left(\tau^{*}-\tau_{\mathrm{tr}}^{*}\right)^{1 / 2}+O\left(\tau^{*}-\tau_{\mathrm{tr}}^{*}\right), \\
X^{*} & \approx \frac{A^{2}}{\left[S_{0}^{*} \beta\right]^{1.5}}\left(\tau^{*}-\tau_{\mathrm{tr}}^{*}\right)^{1 / 2}+O\left(\tau^{*}-\tau_{\mathrm{tr}}^{*}\right), \\
A & =1-k_{d}^{*}-2 k_{d}^{*} S_{0}^{*} .
\end{aligned}
$$

For Case $3\left(\gamma^{-1 / 2}<S_{0}^{*}\right)$, line (c) in Figure 1, a limit point bifurcation occurs at

$$
\tau^{*}=\tau_{\max }^{*}=\frac{(1+2 \sqrt{\gamma}) \beta}{1-(1+2 \sqrt{\gamma}) k_{d}^{*}} .
$$

At this point the no-washout solution branch is given by

$$
\left(S^{*}, X^{*}, \tau^{*}\right)=\left(\gamma^{-1 / 2}, \frac{S_{0}^{*}-\gamma^{-1 / 2}}{\beta+k_{d}^{*} \tau_{\max }^{*}}, \tau_{\max }^{*}\right)
$$

For values of the residence time slightly larger than the value at the bifurcation point, the concentrations along the no-washout branch are given by

$$
\begin{aligned}
S_{-}^{*} & \approx \gamma^{-1 / 2}-\frac{\left[\gamma^{1 / 2} \beta\right]^{1 / 2}}{g \beta} A\left(\tau^{*}-\tau_{\mathrm{tr}}^{*}\right)^{1 / 2}+O\left(\tau^{*}-\tau_{\mathrm{tr}}^{*}\right), \\
X^{*} & \approx \frac{S_{0}^{*}-\gamma^{-1 / 2}}{\beta} A+\frac{A^{2}}{\left[\gamma^{1 / 2} \beta\right]^{1.5}}\left(\tau^{*}-\tau_{\mathrm{tr}}^{*}\right)^{1 / 2}+O\left(\tau^{*}-\tau_{\mathrm{tr}}^{*}\right), \\
A & =1-k_{d}^{*}-2 k_{d}^{*} \gamma^{1 / 2} .
\end{aligned}
$$

3.4.2. Large residence times $(\tau * \gg 1)$ Only the solution branch $S_{-}^{*}$ is meaningful for large residence times. We have

$$
\begin{aligned}
S_{-}^{*} & \approx \frac{1-k_{d}^{*}-A}{2 k_{d}^{*} \gamma}+\frac{1-k_{d}^{*}-A}{2 A k_{d}^{* 2} \gamma} \frac{\beta}{\tau^{*}}+O\left(\frac{1}{\tau^{*}}\right)^{2}, \\
X^{*} & \approx\left\{\frac{S_{0}^{*}}{k_{d}^{*}}-\frac{\left[1-k_{d}^{*}-A\right]}{2 k_{d}^{*^{2}} \gamma}\right\} \frac{1}{\tau^{*}}+O\left(\frac{1}{\tau^{*}}\right)^{2}, \\
A & =\left[\left(1-k_{d}^{*}\right)^{2}-4 k_{d}^{*} \gamma\right]^{1 / 2} .
\end{aligned}
$$


The substrate concentration at high residence times (3.8) is independent of the substrate concentration in the feed $\left(S_{0}^{*}\right)$. For small values of the inhibition constant these simplify to

$$
\begin{aligned}
& S_{-}^{*} \approx \frac{1}{1-k_{d}^{*}}+\frac{2 k_{d}^{*^{2}}}{\left(1-k_{d}^{*}\right)^{3}} \gamma+\left[\frac{1}{\left(1-k_{d}^{*}\right)^{2}}+\frac{3 k_{d}^{*^{2}}}{\left(1-k_{d}^{*}\right)^{4}} \gamma\right] \frac{\beta}{\tau^{*}}+O\left(\tau^{*^{-2}}, \gamma^{2}\right), \\
& X^{*} \approx\left[\frac{S_{0}^{*}}{k_{d}^{*}}-\frac{1}{1-k_{d}^{*}}-\frac{k_{d}^{*^{2}}}{\left(1-k_{d}^{*}\right)^{3}} \gamma\right] \frac{1}{\tau^{*}}+O\left(\tau^{*^{-2}}, \gamma^{2}\right) .
\end{aligned}
$$

This shows the increase (decrease) in substrate (biomass) concentration at high residence times due to small inhibition.

In the following we define the limiting substrate concentration at infinite residence time, $S_{\tau^{*}=\infty}^{*}$. In the case of no substrate inhibition, $\gamma=0$, and infinite residence time,

$$
S^{*}\left(\tau^{*}=\infty, \gamma=0\right)=\frac{k_{d}^{*}}{1+k_{d}^{*}} .
$$

As the value of the substrate inhibition constant $\gamma$ increases from zero, the value for $S^{*}\left(\tau^{*}=\infty, \gamma\right)$ decreases. In the case of small substrate inhibition, $\gamma \ll 1$, and infinite residence time,

$$
\frac{S^{*}\left(\tau^{*}=\infty, \gamma \ll 1\right)}{S^{*}\left(\tau^{*}=\infty, \gamma=0\right)}=1+\frac{k_{d}^{*}}{\left(1-k_{d}^{*}\right)^{2}} \gamma
$$

Thus the proportional increase in substrate concentration at high residence times, compared to a system without substrate inhibition, is independent of the substrate concentration in the feed.

\section{Conclusion}

We have investigated a reactor model for the interaction between a microorganism and a rate-controlling substrate subject to substrate inhibition. Substrate inhibition was modelled using the Andrews function. We considered a generalized reactor model in which the well-stirred flow reactor is a limiting case and included a microorganism decay coefficient.

Models of this form have been comprehensively studied in the mathematics literature for generalized inhibition functions. However, chemical engineers use specific reaction functions to model real systems. Thus we have revisited this problem to fill out some "missing details".

A scaling of the equations reveals that the key dimensionless parameter which controls the degree of inhibition is the quotient

$$
\gamma=\frac{K_{s}}{K_{I}}
$$


Two generic steady-state diagrams exist for this model. For low values of the feed concentration $\left(S_{0}^{*}<\gamma^{-1 / 2}\right.$, curve (a) in Figure 2$)$, there is only ever one stable steadystate solution. Thus the steady-state diagram is similar to those for systems where the specific growth rate function is a monotonic increasing function of the substrate concentration. For high values of the feed concentration $\left(S_{0}^{*}>\gamma^{-1 / 2}\right.$, curve (c) in Figure 2), there is a region of bistability in which the washout solution and the nowashout solution are both locally stable and the ultimate behaviour of the system depends upon the initial conditions.

We generalized earlier findings of Andrews [1] regarding how the critical value of the residence time responds to changes in the substrate concentration and/or the inhibition.

Asymptotic solutions were found in the limit of small and large values of the inhibition constant. Knowledge of the steady-state solutions and their asymptotic limits may be useful to estimate parameter values from experimental data.

\section{Acknowledgement}

M. I. Nelson would like to thank the Faculty of Informatics (University of Wollongong) for financial support that allowed completion of this paper.

\section{Appendix A. Symbols used}

We denote the concentration of the substrate $S$ and the microorganism $X$ by $|S|$ and $|X|$, respectively. Further notation is listed in Table 1 .

\section{Appendix B. Global stability of the washout branch}

Here we obtain some results which ensure that $\lim _{t^{*} \rightarrow \infty} X^{*}=0$, that is, that the "washout solution" $\left(S^{*}, X^{*}\right)=\left(S_{0}^{*}, 0\right)$ is globally stable. We first show that if $k_{d}^{*}$ is greater than some critical value then $\lim _{t^{*} \rightarrow \infty} X^{*}=0$. We then show that when the decay parameter is lower than the critical value we have $\lim _{t^{*} \rightarrow \infty} X^{*}=0$ provided that the residence time $\tau^{*}$ is sufficiently low.

Consider equation (2.5):

$$
\frac{d X^{*}}{d t^{*}}=-\frac{\beta}{\tau^{*}} X^{*}+\left[\mu\left(S^{*}\right)-k_{d}^{*}\right] X^{*}
$$

If $k_{d}^{*} \geq \mu\left(S^{*}\right)$ then

$$
\frac{d X^{*}}{d t^{*}} \leq-\frac{\beta}{\tau^{*}} X^{*}
$$

and it follows that $\lim _{t^{*} \rightarrow \infty} X^{*}=0$.

The maximum value of $\mu\left(S^{*}\right)$ occurs when $S^{*}=S_{\mathrm{cr}}^{*} \gamma^{-1 / 2}$ :

$$
\mu\left(S^{*}\right)_{\max }=\frac{1}{1+2 \sqrt{\gamma}} ;
$$


Table 1.

\begin{tabular}{|c|c|c|}
\hline Symbol & Description & Units \\
\hline$F$ & Flow rate through the bioreactor & $\mathrm{dm}^{3} \mathrm{hr}^{-1}$ \\
\hline$J$ & Jacobian matrix & - \\
\hline$K_{I}$ & Noncompetitive substrate inhibition constant & $|S|$ \\
\hline$K_{s}$ & Monod constant & $|S|$ \\
\hline$S$ & Substrate concentration within the bioreactor & $|S|$ \\
\hline$S^{*}$ & Dimensionless substrate concentration $S^{*}=S / K_{s}$ & - \\
\hline$S_{\mathrm{cr}}$ & $\begin{array}{l}\text { The value of the substrate concentration at which the specific } \\
\text { growth rate obtains its maximum value }\end{array}$ & $|S|$ \\
\hline$S_{\mathrm{cr}}^{*}$ & $\begin{array}{l}\text { The value of the dimensionless substrate concentration at which } \\
\text { the dimensionless specific growth rate obtains its maximum } \\
\text { value }\end{array}$ & - \\
\hline$S_{l}^{*}$ & $\begin{array}{l}\text { Dimensionless substrate concentration at the limit point } \\
\text { bifurcation }\end{array}$ & - \\
\hline$\hat{S^{*}}$ & $\begin{array}{l}\text { Dimensionless substrate concentration along the no-washout } \\
\text { solution branch }\end{array}$ & - \\
\hline$S_{0}$ & Substrate concentration in the feed & $|S|$ \\
\hline$S_{0}^{*}$ & Dimensionless substrate concentration in the feed $S_{0}^{*}=S_{0} / K_{s}$ & - \\
\hline$V$ & Volume of the bioreactor & $\mathrm{dm}^{3}$ \\
\hline$X$ & Concentration of microorganisms within the bioreactor & $|X|$ \\
\hline$X^{*}$ & Dimensionless microorganism concentration $X^{*}=X /\left(\alpha_{s} K_{s}\right)$ & - \\
\hline$k_{d}$ & Decay coefficient & $\mathrm{hr}^{-1}$ \\
\hline$k_{d}^{*}$ & Dimensionless decay coefficient $k_{d}^{*}=k_{d} / \mu_{m}$ & - \\
\hline$k_{d, \max , 1}^{*}$ & $\begin{array}{l}\text { A critical value of the dimensionless decay coefficient } k_{d, \text { max }, 1}^{*}= \\
1 /(1+2 \sqrt{\gamma})\end{array}$ & - \\
\hline$k_{d, \max , 2}^{*}$ & $\begin{array}{l}\text { A critical value of the dimensionless decay coefficient } k_{d, \max , 2}^{*}= \\
S_{0}^{*} /\left(1+S_{0}^{*}+\gamma S_{0}^{*^{2}}\right)\end{array}$ & - \\
\hline$t$ & Time & $\mathrm{hr}^{-1}$ \\
\hline$t^{*}$ & Dimensionless time $t^{*}=\mu_{m} t$ & - \\
\hline$\alpha_{s}$ & $\begin{array}{l}\text { Substrate yield factor, the ratio of the weight of product } \\
\text { produced to the weight of substrate consumed }\end{array}$ & $|X||S|^{-1}$ \\
\hline$\beta$ & Reactor parameter, $0 \leq \beta \leq 1$ & - \\
\hline$\gamma$ & Dimensionless substrate inhibition constant $\gamma=K_{s} / K_{I}$ & - \\
\hline$\mu(S)$ & Specific growth rate function & $\mathrm{hr}^{-1}$ \\
\hline$\mu_{m}$ & $\begin{array}{l}\text { Maximum specific growth rate in the absence of substrate } \\
\text { inhibition }\end{array}$ & $\mathrm{hr}^{-1}$ \\
\hline$\tau$ & Residence time & hr \\
\hline$\tau^{*}$ & Dimensionless residence time $\tau^{*}=V \mu_{m} / F$ & - \\
\hline$\tau_{\max }^{*}$ & $\begin{array}{l}\text { A value of the dimensionless residence time } \\
\tau_{\max }^{*}=\frac{(1+2 \sqrt{\gamma}) \beta}{1-(1+2 \sqrt{\gamma}) k_{d}^{*}}\end{array}$ & - \\
\hline$\tau_{\mathrm{tr}}^{*}$ & $\begin{array}{l}\text { The value of the dimensionless residence time at the } \\
\text { transcritical bifurcation } \tau_{\mathrm{tr}}^{*}=\frac{\left(1+S_{0}^{*}+\gamma S_{0}^{2}\right) \beta}{S_{0}^{*}-\left(1+S_{0}^{*}+\gamma S_{0}^{*^{2}}\right) k_{d}^{*}}\end{array}$ & - \\
\hline
\end{tabular}


see (2.6) and (2.7). Thus the washout solution is globally asymptotically stable if $k_{d}^{*} \geq k_{d, \max , 1}^{*}=1 /(1+2 \sqrt{\gamma})$.

When $S_{0}^{*}<\gamma^{-1 / 2}$ the value of the substrate concentration at which the growth rate function obtains its global maximum is outside the invariant region. The local maximum of the growth rate function within the invariant region is

$$
\mu\left(S^{*}\right)_{\max }=\frac{1}{1+S_{0}^{*}+\gamma S_{0}^{*^{2}}} .
$$

The washout branch is now globally stable if

$$
k_{d}^{*} \geq k_{d, \max , 2}^{*}=\frac{S_{0}}{1+S_{0}^{*}+\gamma S_{0}^{*^{2}}} .
$$

Note that when $S_{0}^{*}=\gamma^{-1 / 2}$ we have $k_{d \text {,max }, 2}=k_{d \text {,max,1 }}$. We now consider the case when $k_{d}^{*}$ is lower than these critical values. Using an identical method it can be shown that:

(i) If $S^{*} \geq \gamma^{-1 / 2}$ and $k_{d}^{*}<k_{d, \text { max, } 1}^{*}=1 /(1+2 \sqrt{\gamma})$ then we have $\lim _{t^{*} \rightarrow \infty} X^{*}=0$ when

$$
\tau^{*}<\tau_{\max }^{*}=\frac{(1+2 \sqrt{\gamma}) \beta}{1-(1+2 \sqrt{\gamma}) k_{d}^{*}} .
$$

This bound is sharp because the lower branch of the no-washout solution $\left(\hat{S}_{-}^{*}\right)$ is locally stable when $\tau^{*}>\tau_{\text {max }}^{*}$.

(ii) If $S^{*}<\gamma^{-1 / 2}$ and $k_{d}^{*}<k_{d, \max , 2}^{*}=S_{0}^{*} /\left(1+S_{0}^{*}+\gamma S_{0}^{*^{2}}\right)$ then we have $\lim _{t^{*} \rightarrow \infty} X^{*}=0$ when

$$
\tau^{*}<\tau_{\mathrm{tr}}^{*}=\frac{\left(1+S_{0}^{*}+\gamma S_{0}^{*^{2}}\right) \beta}{S_{0}^{*}-\left(1+S_{0}^{*}+\gamma S_{0}^{*^{2}}\right) k_{d}^{*}} .
$$

This bound is sharp because (3.4) shows that the washout solution is locally unstable when $k_{d}^{*}<k_{d, \text { cr }}^{*}$ if $\tau^{*}>\tau_{\text {tr }}^{*}$.

\section{Appendix C. Analysis of the no-washout solution branch}

The substrate concentration $\hat{S^{*}}$ satisfies the quadratic equation

$$
\begin{aligned}
\mathcal{G}\left(\hat{S^{*}}\right) & =a \hat{S^{*}}-b \hat{S^{*}}+c=0, \\
a & =\left(\beta+k_{d}^{*} \tau^{*}\right) \gamma>0, \\
b & =\left(1-k_{d}^{*}\right) \tau^{*}-\beta, \\
c & =\left(\beta+k_{d}^{*} \tau^{*}\right)>0 .
\end{aligned}
$$

If $b<0$ then the solutions of (C.1) are non-physical because they are both negative. If $b>0$ then the solutions of (C.1) are both positive. (Recall from (3.2) that a positive 
value for $S^{*}$ does not guarantee that the steady-state solution is physically meaningful.) Thus a necessary condition to have a physically meaningful solution is

$$
b>0, \quad \text { which implies } \tau^{*}>\tau_{b}^{*}=\frac{\beta}{1-k_{d}^{*}}>0 .
$$

(From (3.1) we can assume $1-k_{d}^{*}>0$.)

The discriminant of (C.1) is given by

$$
\begin{aligned}
& \mathcal{D}=b^{2}-4 a c=A_{1}\left(\tau^{*}-\tau_{1}^{*}\right)\left(\tau^{*}-\tau_{2}^{*}\right), \\
& A_{1}=\left[1-(1+2 \sqrt{\gamma}) k_{d}^{*}\right]\left[1-(1-2 \sqrt{\gamma}) k_{d}^{*}\right]>0, \\
& \tau_{2}^{*}=\frac{(1+2 \sqrt{\gamma}) \beta}{1-(1+2 \sqrt{\gamma}) k_{d}^{*}}=\tau_{\max }^{*}>0, \quad \tau_{1}^{*}=\frac{(1-2 \sqrt{\gamma}) \beta}{1-(1-2 \sqrt{\gamma}) k_{d}^{*}}<\tau_{2}^{*} .
\end{aligned}
$$

Hence the discriminant is positive when either $\tau^{*}>\tau_{2}^{*}$ or $\tau^{*}<\tau_{1}^{*}$. Observe that

$$
\tau_{2}^{*}=\frac{(1+2 \sqrt{\gamma}) \beta}{1-(1+2 \sqrt{\gamma}) k_{d}^{*}}>\frac{\beta}{1-k_{d}^{*}}=\tau_{b}^{*} .
$$

Thus when $\tau^{*}>\tau_{2}^{*}$ the solutions of (C.1) are both positive and may be physically meaningful. The case $\tau^{*}<\tau_{1}^{*}$ is only of interest when $0<\tau^{*}<\tau_{1}^{*}$. As $k_{d}^{*}>0$ the conditions for the value of $\tau_{1}^{*}$ to be positive are

$$
1-2 \sqrt{\gamma}>0 \text { and } 1-(1-2 \sqrt{\gamma}) k_{d}^{*}>0 .
$$

Assuming these conditions hold, it follows that

$$
0<\tau_{1}^{*}<\frac{\beta}{1-k_{d}^{*}}=\tau_{b}^{*} .
$$

Thus when $0<\tau^{*}<\tau_{1}^{*}$ the solutions of (C.1) are non-physical because they are both negative.

We conclude that the solutions of (C.1) are positive only when $\tau^{*} \geq \tau_{\max }^{*}$.

$$
\text { Appendix D. To show that } \tau_{\mathrm{tr}}^{*} \geq \tau_{\max }^{*}
$$

We have

$$
\begin{aligned}
\tau_{\mathrm{tr}}^{*} & =\frac{\beta\left(1+S_{0}^{*}+\gamma S_{0}^{2}\right)}{-k_{d}^{*}+\left(1-k_{d}^{*}\right) S_{0}^{*}-k_{d}^{*} \gamma S_{0}^{*^{2}}}=\frac{\beta}{A-k_{d}^{*}}, \\
\tau_{\max }^{*} & =\frac{(1+2 \sqrt{\gamma}) \beta}{1-(1+2 \sqrt{\gamma}) k_{d}^{*}}=\frac{\beta}{B-k_{d}^{*}}, \\
A & =\frac{1}{1+\gamma S_{0}^{*}+\frac{1}{S_{0}^{*}}}=\frac{S_{0}^{*}}{1+S_{0}^{*}+\gamma S_{0}^{*^{2}}}, \quad B=\frac{1}{1+2 \sqrt{\gamma}} .
\end{aligned}
$$


The question is only meaningful when both quantities are positive. Thus we may assume that $A>k_{d}^{*}$ and $B>k_{d}^{*}$. The statement follows if we can show that $B \geq A$.

The number $A$ is a particular value of the function

$$
\mu\left(S^{*}\right)=\frac{S^{*}}{1+S^{*}+\gamma S^{*^{2}}} .
$$

The maximum value of this function occurs when $\gamma=S_{0}^{*^{-2}}$ and is

$$
\mu\left(S^{*}\right)_{\max }=\frac{1}{1+2 \sqrt{\gamma}}=B .
$$

It follows that $A=B$ when $\gamma=S_{0}^{*^{-2}}$; otherwise $B>A$. Thus $\tau_{\mathrm{cr}}^{*} \geq \tau_{\max }^{*}$.

\section{References}

[1] J. F. Andrews, "A mathematical model for the continuous culture of microorganisms utilizing inhibitory substrates", Biotechnol. Bioeng. 10 (1968) 707-724; doi:10.1002/bit.260100602.

[2] A. W. Bush and A. E. Cook, "The effect of time delay and growth rate inhibition in the bacterial treatment of wastewater", J. Theoret. Biol. 63 (1976) 385-395; doi:10.1016/0022-5193(76)90041-2.

[3] G. J. Butler and G. S. K. Wolkowicz, "A mathematical model of the chemostat with a general class of functions describing nutrient uptake", SIAM J. Appl. Math. 45 (1985) 138-151; doi:10.1137/0145006.

[4] F. Costa, C. Quintelas and T. Tavares, "Kinetics of biodegradation of diethylketone by Arthrobacter viscosus", Biodegradation 23 (2012) 81-92; doi:10.1007/s10532-011-9488-7.

[5] C. N. Economou, G. Aggelis, S. Pavlou and D. V. Vayenas, "Modeling of single-cell oil production under nitrogen-limited and substrate inhibition conditions", Biotechnol. Bioeng. 108 (2010) 1049-1055; doi:10.1002/bit.23026.

[6] B. Fiedler and S.-B. Hsu, "Non-periodicity in chemostat equations: a multi-dimension negative Bendixson-Dulac criterion", J. Math. Biology 59 (2009) 233-253; doi:10.1007/s00285-008-0229-4.

[7] D. Huang and J. G. Becker, "Dehalorespiration model that incorporates the self-inhibition and biomass inactivation effects of high tetrachloroethene concentrations", Environ. Sci. Technol. 45 (2011) 1093-1099; doi:10.1021/es102729v.

[8] D. W. Jordan and P. Smith, Nonlinear ordinary differential equations, 2nd edn (Clarendon Press, 1989).

[9] B. Li, "Global asymptotic behaviour of the chemostat: General response functions and different removal rates", SIAM J. Appl. Appl. Math. 59 (1998) 411-422; doi:10.1137/S003613999631100X.

[10] T. Sari, "Lyapunov function for the chemostat with variable yields", C. R. Math. 348 (2010) 747-751; doi:10.1016/j.crma.2010.06.008.

[11] T. Sari and F. Mazenc, "Global dynamics of the chemostat with different removal rates and variable yields", Math. Biosci. Engng. 8 (2011) 827-840; doi:10.3934/mbe.2011.8.827.

[12] L. Wang and G. S. K. Wolkowicz, "A delayed chemostat model with general nonmonotone response functions and differential removal rates", J. Math. Anal. Appl. 321 (2006) 452-468; doi:10.1016/j.jmaa.2005.08.014.

[13] G. S. K. Wolkowicz and Z. Lu, "Global dynamics of a mathematical model of competition in the chemostat: General response functions and differential death rates", SIAM J. Appl. Math. 52 (1992) 222-233; doi:10.1137/0152012. 